\title{
Vivamos a la vista de todos'
}

Let us be seen by all

\author{
LUIS BRANDA \\ Universidad de Girona \\ España \\ luis.branda@udg.edu
}

Resumen. Desde ya hace años se ha tratado de evitar y corregir el comportamiento no profesional como asimismo los errores resultantes de la práctica médica. La literatura sobre lo que se ha llamado profesionalismo es extensa, incluyendo la narrativa de historias sobre la violación de esos valores que deben guiar la práctica médica. Las expectativas del comportamiento médico deben ser altas pero también debe haber comprensión a los fallos del cumplimiento de esas expectativas. El proceso de sanar debe involucrar la identificación por el profesional del daño que ha causado a otros (rendición de cuentas) y un genuino arrepentimiento que debe llevar a la posibilidad del perdón - perdón, no en un sentido religioso sino en el contexto social de la interacción entre aquellos que causan daño y los que han sido dañados -. El desafío a las instituciones académicas es el incluir en la formación del médico el desarrollo de actitudes y valores profesionales.

Palabras clave: Profesionalismo, errores médicos, valores, actitudes, formación médica.
Summary. For many years attempts have been made to avoid and correct unprofessional behavior and errors in medical practice. The literature on what has been called professionalism is extensive, including narratives on the breaching of those values that guide the practice of medicine. Expectations for professional behaviour must be high, but there should also be an understanding of the failure to meet those expectations. The healing process should include the identification by the professional of the harm that he or she has done to others (accounting for your actions) and a genuine repentance which leads to the possibility of forgiveness - forgiveness, not in a religious sense - but in the social context of interaction between those who have caused harm and those who have been harmed.The challenge to academic institutions is to include in their curricula the development of attitudes and professional values.

Key words: Professionalism; medical errors; values; attitudes; medical curriculum; literature.

\footnotetext{
${ }^{\text {I }}$ Para citar este artículo: Branda, L., Vivir a la vista de todos. Álabe 4, diciembre 2oIr [http://www.ual.es/alabe]
} 
Todos aquellos que están interesados en las competencias que el médico debe poseer en el área de valores profesionales y actitudes, es probable que hayan acumulado un pequeño montón de piedras de la enorme cantera que constituye la literatura sobre el profesionalismo. Este artículo agrega algunos granos de arena, que más que ideas, son reflexiones, elucubraciones y especulaciones en relación a donde se está o se debe estar en el área del profesionalismo médico.

Una constante preocupación ha sido identificar los valores y actitudes que los médicos deben poseer. Ya en el siglo XVI dos escritores españoles, Alfonso de Miranda en I562 y Enrique Jorge Enríquez en I595 publicaron diálogos que tratan sobre las características que el médico debe poseer (Miranda, I983; Enríquez, I595). El resumen que hace Lee, basado ambas obras, de esas características es el siguiente:

Ser humano, manso, humilde, no soberbio ni vanaglorioso, caritativo con los pobres, afable, de buena memoria, temeroso de Dios, no vengativo, saber guardar secretos, no murmurador ni envidioso, modesto, prudente, templado, no demasiado osado, firme en sus obras, bien afortunado en sus curas, pacífico, benigno, honesto, recogido, dado a las letras, curioso, no ocioso, no dado a los naipes, ducho en las doctrinas de médicos antiguos y modernos, moderado en el comer, celoso de su honra y de buen linaje, que no huya de las disputas sobre cosas de su ciencia, haber estudiado fuera de su patria por largo tiempo, que tenga buena memoria, buen juicio, buen rostro, ser buen retórico, vestirse decente, limpio, templado en el comer, que no ande de convites, tener la confianza de los enfermos. (Lee, 2OII)

Desde hace mucho tiempo es aceptado que varias de estas características, aunque no todas, deben estar presente, incluyendo honestidad, actuando con integridad, dedicación a satisfacer las necesidad de los pacientes, reducir las barreras para un cuidado de salud equitativo y atenerse a un código de conducta ético. La mayor dificultad, en lo que se ha llamado profesionalismo, se relaciona a la identificación y definición de esos valores y actitudes, y de qué manera se puede demostrar que se poseen.

Existen varias definiciones de lo que es profesionalismo médico. Albert Oriol lo define como

el ideario que dicen profesar los médicos, que guía su actuación, y se expresa en forma de conductas características (profesionalidad). El profesionalismo es el conjunto de valores y compromisos por los que la sociedad les otorga a los médicos un contrato social específico y los reconoce como profesión. El compromiso vinculante de los médicos se expresa en documentos como el Juramento Hipocrático desde hace más de dos milenios y que actualmente están en proceso de actualización para adaptarlos a la sociedad contemporánea, tanto en su formulación como en el contenido, incorporando valores sociales que en sus orígenes no estaban explicitados. (Oriol, 2OIO)

En un breve artículo publicado ya hace muchos años, pero que sigue teniendo actualidad, Crawshaw y colaboradores reafirman la obligación de medicina de proporcio- 
nar un cuidado basado en los mejores intereses del paciente (Crawshaw, et al. r995). Este artículo fue otra de las llamadas de atención al riesgo que enfrenta la profesión médica de perder de vista que la medicina está fundamentada en ciertos valores profesionales. El artículo señala que

By its tradition and very nature, medicine is a special kind of human activity -one that cannot be pursued effectively without the virtues of humility, honesty, intellectual integrity, compassion, and effacement of excessive self-interest. This traits mark physicians as members of a moral community dedicated to something other than its own self-interest. (I995: I563)

En el primer capítulo de su excelente libro que enfoca como evaluar los valores y actitudes del médico, Measuring Medical Professionalism, David Stern (2006) cuenta una historia que probablemente sea ya bastante conocida.

A pious man was standing in a long line at the gates of heaven, waiting for his audience with St. Peter. After one hour, a man in a white coat and stethoscope walks up to the front of the line, past St. Peter, and right into heaven.

The pious man upset about the breach in protocol, asked St. Peter, "Why did that doctor skip the line and go right in?" "Oh, him?" said St. Peter, "That's God -but he just thinks he's a doctor.” (Stern, 2006: 3 )

La relación entre un comportamiento divino y el de los médicos ha sido un tema bastante trillado en la narrativa médica. Un ejemplo bien ilustrativo lo es el libro de Robert Marion, Learning to play God (1992). En el estilo de lo que podría llamarse un dietario, Marion describe sus experiencias desde su ingreso como estudiante de grado de medicina, motivado por idealismo y compasión hasta su período como residente en pediatría forzado por la realidad sanitaria de tomar decisiones más allá de lo que es razonable esperar de un profesional que se encuentra todavía en formación.

Medical education in the United States today takes people who enter the system filled with humanism and idealism and ultimately forces them to surrender these ideals by the very process that turns them into technically competent and intellectually capable physicians. (Marion I992: 262)

En otro libro, Letters to a Young Doctor, Richard Selzer narra sus experiencias como médico, incluyendo algunas, como la titulada, Letter to a young surgeon, donde escribe lo que le dice a un medico novel: "All right. You fainted in the operating room... you are making altogether too mucho of it. You have merely announced your humanity. Only de gods do not faint..." (1996: 55).

Se dice que Alejandro Magno era acompañado en su carro de combate por un esclavo que constantemente le recordaba su mortalidad susurrándole: memento mori; qui- 
zás este recordatorio de ser mortal es recomendable a todos los que poseen cierto grado de influencia, incluyendo los médicos.

Las expectativas del comportamiento del médico son altas; se requiere que demuestre compasión y respeto por los pacientes, incluso aquellos muy difíciles. Sin embargo, muy a menudo hay poca comprensión de o tolerancia a los fallos de cumplimiento de esas expectativas. Es razonable pensar que durante mucho tiempo los médicos se han esforzado por mantener altos estándares de comportamiento profesional, pero como seres humanos son por lo tanto falibles.

Los resultados de los estudios de Danziger y colaboradores sobre como se llevan a cabo decisiones judiciales, Extraneous factors in judicial decisions (2OII) refuerzan la evidencia ya existente sobre la susceptibilidad de jueces con extensa experiencia a sesgos psicológicos y a otros factores extraños como lo son las estrategias de simplificación en casos complejos (Vidmar, 2OII). Aunque el foco de los estudios de Danziger et al. han sido las decisiones legales, los autores concluyen que la presencia de algunas de estas estrategias son operativas en otro tipo de decisiones como lo son las médicas. Jueces y médicos se suponen que están entrenados a basar sus decisiones en los hechos, pero son también humanos y por lo tanto susceptibles a todo tipo de sesgos cognitivos.

Entre los valores que se identifican parte del profesionalismo se ha incluido, como uno fundamental para la profesión médica, la empatía (Hojat, M. et al. 2002) definida como la habilidad de comprender las perspectivas de otra persona y sus sentimientos, sin sentirse demasiado implicado emocionalmente. La empatía es un concepto que involucra ambos ámbitos, el cognitivo como el afectivo. En el ámbito cognitivo la empatía se ha descrito como la habilidad de comprender lo que ha sentido, conocido o presenciado alguien y poder ver las cosas desde el punto de vista de otra persona. En el ámbito afectivo la empatía implica la capacidad de poder integrar en uno mismo los sentimientos de otra persona pero manteniendo una distancia que le permite a la persona mantener su propia identidad. Esto último se refiere más a la simpatía que a la empatía.

En su artículo sobre este tópico, Charles Aring ha señalado la importancia para el médico de comprender la diferencia entre la simpatía y la empatía.

Just this much may save the physician from acting in a manner suggesting that he himself imposed the disease on his patient and, while it should never lift him in any sense above de misery and suffering, it may allow him a certain freedom to act for the patient's greatest good. The ultimate test of any medical endeavor is what it does for the patient. (Aring, $195^{8:} 45^{2}$ )

También debe reconocerse que existe variabilidad en los niveles de empatía de las distintas personas, incluyendo los miembros de la profesión médica. En su libro, The Science of Evil, Simon Baron-Cohen describe siete niveles empatía de las personas en relación al daño inferido a otras dentro de una distribución que el autor llama The Empathy Bell Curve. (20I: I9) En el nivel o, la persona tiene una ausencia total de empatía. Cuando a estas personas se les indica que han dañado a otros, esto no tiene ningún signi- 
ficado y no pueden tener sensaciones de remordimiento o culpabilidad desde que no pueden entender como se siente las otras personas. Al nivel I corresponden a las personas que todavía son capaces de hacer daño a otros, pero en cierto grado pueden reflexionar en lo que han hecho y sentir cierto remordimiento. Sin embargo no pueden controlar el comportamiento que resulta en un daño o injuria a otros. En el nivel 2 se encuentran las personas que todavía tienen dificultades importantes con la empatía, pero pueden vislumbrar cómo otros se sienten y esto los inhibe en cierto modo en la agresividad de su comportamiento. En el nivel 3 las personas saben que tiene dificultades con la empatía y tratan de enmascarar o compensar esta deficiencia. Esto lo hacen quizás evitando trabajos o relaciones personales donde se requiere una demanda constante por empatía. Las personas en el nivel 4 tienen un grado de empatía debajo de la media. En general esto no tiene un efecto importante en el comportamiento diario aunque estas personas se sienten más cómodas cuando la conversación es sobre temas que no se relacionan a las emociones. En el nivel 5 las personas tienen un grado de empatía marginalmente superior a la media. Aunque las personas a este nivel no están pensando constantemente de cómo se sienten otros, son muy cuidadosas en hacer valer su opinión para no dominar o entrometerse. Y, finalmente, en el nivel 6 se encuentran personas con un extraordinario nivel de empatía, cuyo comportamiento está fundamentalmente centrado en tener en cuenta los sentimientos de otros y hacen un enorme esfuerzo para dar apoyo.

En una de las historias relatadas por Selzer en su libro, titulada Brute (I996: 59) el autor relata su propia violación de algunos valores profesionales, violación que el autor reconoce ser el resultado de sesgos sociales y culturales.

El libro de Baron-Cohen es un análisis de situaciones de crueldad que no se han demostrado habituales en la práctica de la profesión médica, pero la relación que este autor presenta del grado de empatía con esas situaciones puede considerarse aleccionadora. En relación con el nivel o ("zero negree of empathy") Baron-Cohen señala que

Zero degrees of empathy means you have not awareness of how you come across to others, how to interact with others, or how to anticipate their feelings or reactions. Your Empathy Mechanism functions at Level o. You feel mystified by why relationships don't work out, and your lack of empathy creates a deep-seated self-centeredness. Other people's thoughts and feelings are just off your radar. This leaves you doomed to do your own thing, in your own little bubble, not just oblivious to others people's feelings and thoughts but also oblivious to the idea that there might even be other points of view. The consequence is that you believe Ioo percent in the rightness of your own ideas and beliefs, and judge anyone who does not hold your beliefs as wrong or stupid. (2OII: 43)

El filósofo vienés, Martin Buber, en su famoso libro Ich und Du (Yo and Tu) (Buber, I995), contrasta el yo-tú modo de ser, donde la relación con otra persona es un fin por sí mismo, con el I-ello donde la relación con una persona es con el fin de utilizarla; Buber argumenta que este último modo de tratar a una persona la devalúa. 
Esta es, empero, la sublime melancolía de nuestro destino: que todo Tú haya de convertirse en un Ello en nuestro mundo. Por muy presente en exclusiva que hubiese estado en la relación inmmediata, tan pronto como ésta se ha agotado o ha sido contaminada de mediadez, el Tú deviene un objeto entre objetos, quizás el objeto más sobresaliente, pero un objeto más, fijado según medida y límites. (Buber, I995: I7).

En su discurso de investidura como Doctor Honoris Causa de la Universitat de Barcelona, el profesor Claudio Magris hace referencia a su libro Le Voci, donde describe como

un hombre se enamora de las voces grabadas en los contestadores automáticos, y entonces les llama, pero sólo cuando las mujeres de carne y hueso no están en casa; habla con voces registradas, las mujeres se convierten en puros números de teléfono. (20II: 77)

Los sujetos se han convertido en objetos.

De acuerdo a Baron-Cohen,

When our empathy is switched off, we are solely in the "I" mode. In such a state we relate to things or to people as if they were just things. Treating other people as if they were just objects is one of the worst things you can do to another human being, to ignore their subjectivity, their thoughts and feelings. (Baron-Cohen, 2OII: 7-8).

Buber ya lo había dicho de modo inequívoco, "En fin, con toda la seriedad de la verdad, escucha esto: sin el Ello no puede vivir el ser humano. Pero quien solamente vive con el Ello no es ser humano.” (I995: 29)

En un diálogo del poema épico el Bhagavad-Gita, Arjuna, el hijo del Rey Pandu, le dice a Krisha, el líder del clan indio de los Vrishni: “¡Oh, Krisha! ¿Qué es lo que le impele a uno a los actos pecaminosos, aun involuntariamente como si se le obligara a la fuerza?" (Bhagavad-Gita 3:36). Lucius Annaeus Séneca describe en sus Epístolas morales a Lucilio (Séneca, I986) la lucha tenaz de controlar los impulsos irracionales y ser capaz de actuar siendo consistente en mantener el concepto de integridad.

Todo esto debe considerarse como recordatorio para cuando nos enfrentamos con una violación del comportamiento profesional, no para justificarlo, pero para comprenderlo y así poder comenzar el proceso de corrección para el futuro en forma productiva.

Hay muchas maneras por las cuales la violación de valores profesionales puede identificarse, discutirse y corregirse, comenzando con la auto-revelación de esa violación. El informe del Institute of Medicine (IOM) sobre errores médicos (Kohn, L. T.; Corrigan, J. M. y Donalson, M. S. Eds. 2OOI), ha tenido un efecto beneficioso fomentando el desarrollo de mecanismos para controlar y reducir esos errores. El título del informe 
del IOM, To err is human es sacado del poema de Alexander Pope, An essay on criticism. (Pope, I858). El verso completo del poema es: "To err is human, to forgive, Divine." (I858: 2I3) Aunque to forgive no es parte del título del libro del IOM, es razonable el establecer una relación entre un error que tiene el resultado de un daño no intencional y lo que debe pasar luego: identificación y declaración del error; arrepentimiento, es decir, el pesar de haber hecho algo dañoso; la compensación a la persona dañada; y el perdón. El último verso del poema de Pope es significativo: "Not free from Faults, nor yet too vain to mend." (I858: 22I)

La ética de perdonar no significa que la persona dañada resuelve el problema al perdonar a la persona responsable por el daño, pero plantea preguntas como las que hace Nancy Berlinger en su libro After Harm.

What are the words and actions of the individuals and what are the policies and practices of the institution, that if completed in the aftermath of medical harm, may offer injured patients and their families the possibility of forgiving those responsible for the harm? (Berlinger, 2005:)

El eticista Dietrich Bonhoeffer en Cartas de amor desde la prisión (1998) plantea algunas cuestiones que son relevantes a la pregunta de Berlinger. Lo que plantea Bonhoeffer incluye preguntas como: ¿Qué significa decir la verdad? ¿Quién sufre como resultado del daño? ¿El paciente? ¿La familia? ¿Cuáles son las responsabilidades hacia aquellos que han sufrido como resultado del daño no intencional que han recibido? ¿Cuál es la naturaleza de la relación entre un paciente que ha sufrido daño y el médico? ¿Cuál es la naturaleza de la relación entre un paciente que ha sufrido daño y la institución donde el daño a ocurrido? ¿Como podemos evitar la tentación de suponer que seremos perdonados por nuestros errores en lugar de comportarse de tal manera que hace posible ser perdonados por aquellos que hemos hecho sufrir?

Las respuestas a las preguntas previas no es fácil de encontrar, pero se puede comenzar analizando el concepto de perdón. El concepto de perdón, no en un enfoque religioso sino en el contexto social de la interacción entre aquellos que causan daño y los que han sido dañados.

En su libro, The cost of discipleship, Bonhoeffer acuña la frase “cheap grace” y argumenta en contra el perdonar sin arrepentimiento y disciplina para promover el cambio sostenible en el comportamiento profesional (Bonhoeffer i964). En una de sus Epístolas, titulada Hemos de reconocer los defectos y confiar en corregirlos, Séneca comenta sobre el camino a seguir para una mejora personal reconociendo en forma honesta que la fuente de nuestros errores está en nosotros mismos:

Y ¿qué otra cosa haces sino mejorarte de día a día, abandonar algunos de tus errores, darte cuenta que están en ti los defectos que piensas que están en las cosas? De hecho atribuimos al lugar y al tiempo algunos de nuestros defectos, mas éstos a cualquier sitio que nos traslademos nos han de acompañar. (Séneca, I989: L, 2, 292) 
¿Por qué nos engañamos? Nuestro mal no procede del exterior; se halla dentro de nosotros, radica en nuestras mismas entrañas y la causa de que difícilmente alcanzamos la salud está en desconocer que padecemos la enfermedad. (Séneca, I989: L, 4, 293)

Pope, en el mismo poema citado previamente, reafirma la necesidad de hacer un examen de nuestros errores:

Who, if once wrong, will needs be always so;

But you, with pleasure own your errors past,

And make each day a critic of the last. (I858: 215)

En esta misma línea de pensamiento, Montaigne en sus Ensayos - ese examen de conciencia que describe más al hombre privado que al hombre público - habla sobre el arrepentimiento diciendo que "No conozco arrepentimiento superficial, mediocre o aparente. Me ha de llegar a todas partes, para llamarlo así, y agarrotarme y afligirme las entrañas..." (Montaigne, 2003: 794). Y, en otra parte dice que "Si se pusiera el arrepentimiento en la balanza habrá de pesar más que el pecado.” (2003: 794). Agrega que el acusar a su fortuna y no a sus actos no se llama arrepentimiento.

Montaigne también dice que,

La manera más común de ablandar los corazones de aquellos a los que hemos ofendido, cuando por tener en sus manos la venganza nos tienen a su merced, es la de moverlos por nuestra sumisión a conmiseración y a piedad. Sin embargo, la bravura y la fortaleza, medios diametralmente opuestos, sirvieron a vedes este mismo efecto." (2003: 5I)

El reconocer los errores es, sin duda una manifestación de bravura y fortaleza que debe acompañarse de solicitar una remisión de la ofensa recibida. El declarar o revelar lo ocurrido es el primer paso en tratar de encontrar una respuesta a las cuestiones previamente señaladas.

Aunque probablemente correctas, aseveraciones defensivas como "siempre se comenten errores" o "todo depende de como se defina error", no conducen a las posibles correcciones en el futuro y no toman en cuenta el rol del paciente como copartícipe en el diálogo de sanar.

Existe un factor que complica este proceso. Entre los médicos es frecuente creer que es casi imposible ser perdonado por un paciente o su familia luego de haber causado daño.

Esto recuerda el intercambio de Dr. Faustos con sus colegas académicos, cuando en su desesperación, asevera, erróneamente que sus ofensas nunca serán perdonadas.

En una entrevista con el boletín médico, Second Opinion, David Hilfiker, el autor de los libros Not all of us are saints y Healing the wounds, le dice al entrevistador: 
What we want for our personal physician is somebody who knows what he is doing and is a top notch expert in whatever field we've got our problem in. Faced with that pressure, it takes enormous courage to say, "I am not what you want. If you want somebody infallible, you're going to have to go some place else, because these are the mistakes I've made, and ...you might be the next one; we must recognize the shame, guilt, and anxiety that evoke the breaches of the expectations [of the patient]. (Hilfiker 1989)

Aunque es aceptable que el médico hable o escriba sobre el "paciente difícil” ha sido menos aceptado manifestar aversión o desprecio flagrante por los pacientes bajo su cuidado. Algunos médicos, sin embargo, han hecho público estos sentimientos a pesar de la larga tradición de mantener lo no-mencionable en familia.

David Hilfiker publicó un artículo que creó mucha controversia, Facing our Mistakes, en el cual hizo público varios de sus errores médicos (Hilfiker, I984). El artículo permitió reflexionar sobre la inevitabilidad de cometer errores, la agonía que esto crea en el profesional y nuestra inhabilidad para enfrentarlos. Se acepta que la mayoría del daño que causan los errores médicos no son el resultado del comportamiento de "malos" médicos. Se ha evidenciado que la mayoría de los médicos sienten un genuino remordimiento, incluso angustia, cuando se dan cuanta que sus acciones han resultado en daño a un paciente que está bajo su cuidado.

El Program in Narrative Medicine del College of Physicians and Surgeons at Columbia University, en Nueva York, ofrece varias actividades educacionales entre ellos los Narrative Medicine Rounds. En estas sesiones los estudiantes de la práctica clínica cuentan sus historias de como han confrontado las crisis éticas durante su formación médica. Estas historias se cuentan desde la perspectiva personal, dejando de lado el plural impersonal de las revistas que han introducido secciones dedicadas a declarar errores médicos. En los Narrative Medicine Rounds, los estudiantes confiesan las dudas que han tenido, los errores que han cometido y a veces, incluso el daño que han hecho.

En el año I982, se inició en Gran Bretaña un proyecto auspiciado por las especialidades de cirugía y anestesia. Se llamó el Confidential Enquiry into Perioperative Deaths (CEPOD). Este proyecto condujo a la creación del National Confidential Enquiry into Patient Outcome \& Death. Desde su creación, el NCEPOD ha publicado cada año alrededor de 65 oo informes que contienen casos en los cuales se analiza las intervenciones sobre pacientes, incluyendo los errores cometidos.

El origen de estos proyectos de evaluación de la calidad del cuidado de salud podría atribuirse a la idea del End Result System originada por Ernest Amory Codman. En un artículo escrito por Avenis Donabedian se pueden encontrar los detalles de la importante contribución que hizo Codman a lo que quizás son los aspectos más problemáticos del sistema de cuidado de la salud, incluyendo la rendición de cuentas al público (Donabedian, I989). Codman publicó un informe muy controvertido sobre los estándares mínimos para los servicios en los hospitales, promoviendo que la práctica de cirugía sea estandardizada. La idea detrás del End result system es que cada hospital haga un segui- 
miento de cada paciente que trata, un seguimiento lo suficiente continuado para poder evaluar si el tratamiento ha sido exitoso o no, y si no lo ha sido el poder hacer correcciones para futuros casos. A su ingreso cada paciente tendría una ficha personal (end result card) donde, en forma breve se registrarían los síntomas, el diagnóstico, el tratamiento, las complicaciones que ocurrieron en el hospital, el diagnóstico al habérsele dado de baja, y el resultado luego de una año de habérsele dado de baja en el hospital. Codman también proponía que cada hospital tuviera un comité que revisara las fichas de cada paciente (efficiency comittee). Este comité examinaría los resultados y determinaría las causas de resultados no satisfactorios para desarrollar mecanismos correctores de esos resultados no exitosos. La clasificación de Codman con respecto a las causas de fracaso incluían la falta de competencias técnicas y de diagnóstico. Algunas de ellas Codman las consideraba atribuibles al médico, algunas al hospital, algunas al paciente y las circunstancias de su enfermedad; algunas se consideraban eran remediables, mientras otras no.

El sistema de Codman establecía un claro mecanismo de rendición de cuentas, no solo por el médico en forma individual, pero también por la institución, sobre el cuidado que proporcionan. Codman lo sintetiza diciendo:

My idea is that the Hospital es a place for mutual help. The patient, the Student, the Profession, the Chief of Service, the Trustee, the Community, and the World-wide Medical Science -each are part owners of "the case". (Donabedian, I989: 245)

Los valores del profesionalismo, por lo tanto, no solo se aplica a los profesionales individualmente, sino que también se aplica a las instituciones académicas y sanitarias las cuales deben aceptar el desafío que presenta el rendir cuentas por sus actuaciones. En relación a la reacción a este desafío, es relevante tener en cuenta lo que le pasó a Codman como resultado de su idea revolucionaria.

El 8 de enero de I9I5, Codman dio una charla sobre el End Result sistem en una reunión llevada a cabo en la Boston Medical Library. En esa reunión Codman proyectó un dibujo suyo donde caricaturizaba al famoso y aún poderoso Massachusetts General Hospital y sus médicos. En esa caricatura se ilustraba una avestruz que comía falsedades y ponía huevos de oro para los médicos de la ilustre área de Back Bay (etiquetados en la caricatura como pertenecientes a el "Death Bed Team", "Surgical and Humbug Team" y "Pediatric and Humbug Team"). La mesa o tarima denominada MGM Clinical Truth, muestra a los administradores del hospital diciendo "If we let her know the truth about our patients would you suppose she would still be willing to lay golden eggs" El Presidente (Rector) de Harvard, Abbott Lowell - quien no estaba presente en la charla de Codman - se muestra en la caricatura, sobre el Charles River diciendo: "I wonder if clinical truth is incompatible with medical science. Could my clinical professors make a living without humbug?" Esta reunión le costó a Codman el ser expulsado del MGH y su ostracismo de la comunidad médica.

Un forum para revelar información sobre errores médicos lo proporcionan las Morbidity and Mortality (M\&M) conferencias que se realizan en los hospitales. La crí- 
tica que se ha hecho a las M\&M conferencias es que el uso de detalles técnicos y la voz pasiva utilizada en las reuniones dispersa la responsabilidad. Sin embargo, el enorme valor de las M\&M conferencias es que proporciona un forum para el auto-criticismo como también para una detallada descripción de posible mecanismos para gestionar el error médico y en forma colegiada la aplicación de estándares.

La limitación real de las M\&M conferencias es que excluyen a la parte perjudicada - el paciente - quién no tiene rol ni representación dentro de este ritual privado y no puede contar con que se haga justicia. Esto no significa que los perjudicados deben necesariamente estar incluidos en las M\&M conferencias, pero que un mecanismo adecuado debe estar disponible a ellos para completar el proceso de sanar a través de la admisión de error y últimamente el perdón. Quizás es más apropiado hablar, no de perdón sino de compasión, con el significado de sufrimiento compartido.

Se ha visto en los últimos años un incremento en los litigios por mala praxis médica. Pero, como lo señala un artículo sobre este tema, los litigios por mala praxis médica, con poca frecuencia compensa a los pacientes lesionados por la negligencia médica y rara vez se identifica, y se le hace rendir cuentas a los proveedores de la atención médica deficiente. (Russell Localio, A. et al. I99I)

No es infrecuente que los médicos cuenten errores que cometieron en el manejo de pacientes, algunos de ellos con resultados catastróficos. El disturbio emocional, esa mezcla de remordimiento, ira, culpa, miedo y sentirse incapaz, constituyen el proceso del duelo. Nuestra ideología nos fuerza a controlar el duelo en las realidades privadas de culpabilidad y dolor. Pero, como Elizabeth Kübler-Ross y otros han enfatizado, la experiencia de duelo se hace soportable y significativa aceptando que del duelo surge lo nuevo. En otro de los relatos que se encuentran en el libro de Selzer éste describe las dificultades de aceptar la fase terminal de un paciente. En esta historia titulada Mercy, un paciente con cáncer terminal llega al hospital donde nada puede hacerse para prolongar su vida y lo que resta es el uso de narcóticos para calmar su dolor. El Dr. Selzer sale de la habitación del paciente y se encuentra con la familia a la que le dice: "He didn't die. He won't... or can't." La familia permanece en silencio y el médico insiste: "He isn't ready yet." A lo cual una anciana le replica: “He is ready. You ain’t." (Selzer, I996: 74).

En el Nuevo Testamento, el Evangelio según San Juan (5. I-I8) cuenta la historia del ángel que aparecía en la fuente de Bethesda en el mercado de Jerusalén. El Ángel de Bethesda se ha simbolizado en la magnífica escultura hecha por Emma Sttebings en i868 que se encuentra en el Central Park en Nueva York. El Evangelio relata que un ángel bajaba en cierta época del año y agitaba las aguas de la fuente del mercado. Quién primero entraba en las aguas se curaba de cualquier enfermedad que padeciera.

En su breve obra de teatro, The Angel that Troubled the Water, Thornton Wilder cambia el final de la parábola. La obra de teatro cuenta de un médico que viene a la fuente de Bethesda con la esperanza de ser el primero en entrar y de esta manera curarse de su sufrimiento. 
El médico le dice al ángel: "My work grows faint." "Heal me that I may continue... let me begin again without this fault that bears me down.” El ángel le cierra el paso al médico y le dice: "Draw back physician, this moment is not for you," "Healing is not for you."

Pero, el médico insiste:

Surely, O Prince, you are not deceived by apparent wholeness. Your eyes can see the nets in which my wings are caught; the sin into which all my endeavours sink halfperformed cannot be concealed from you. (I928: I05)

Y el ángel le responde:

Without your wound where would your power be? It is your very remorse than makes your low voice tremble into the hearts of men. The very angels themselves cannot persuade the wretched and blundering children on earth as can one human being broken on the wheels of living....only the wounded soldiers can serve. Draw back. (I928: Io6)

Las universidades se han estado haciendo una persistente pregunta ¿Se pueden enseñar los valores profesionales y el reconocer como el comportamiento que viola el profesionalismo puede ser identificado y corregido?

La transformación de jóvenes con talento y vocación en profesionales médicos competentes es una tarea educativa complicada que ocupa por lo menos toda una década, mientras que el mantenimiento de la capacidad y la mejora permanente de la competencia de los profesionales de la medicina dura tanto como su actividad profesional, es decir, más de tres décadas. (Oriol, 20IO: $5^{2}$ )

Si la profesionalidad es la expresión conductual de la adherencia a las virtudes y compromisos con el profesionalismo médico, ésta deberá ser considerada por los educadores como una competencia que necesariamente deben ir alcanzando a lo largo del proceso educativo, para luego mantenerla/mejorarla durante toda su vida activa.

(Oriol, 2OIO: 55)

En una era donde se ha reconocido lo obvio, es decir, que la medicina debe estar centrada en el paciente, se espera que las instituciones académicas encuentren maneras creativas de integrar en lo que ya es un currículo atiborrado el desarrollo de habilidades y actitudes que ponen énfasis en el profesionalismo.

Como en casi todas las áreas de la enseñanza, en la educación médica existe una mucha frustración resultante de no tener el tiempo y la oportunidad para transmitir lo que se considera es lo más importante; esto implica que sabemos que es lo más importante en la formación del médico. Cabe hacerse una última pregunta, ¿no es el desarrollo de las actitudes y los valores profesionales lo más importante en la formación del profesional médico? 


\section{BIBLIOGRAFÍA}

-Aring, C. D. (1958). Sympathy and Empathy. Journal of the American Medical Association, I67, 448-452.

- Bahavada-Gita, en español, obtenido el 20 de abril de 20 II desde http://www.bhagavadgita.org/index-spanish.html

- Baron-Cohen, S. (20I). The Science of Evil. On Empathy and the Origins of Cruelty. New York: Perseus.

- Belinger, N. (2005). After Harm: medical errors and the ethics of forgiveness. Baltimore: The John Hopkins University Press.

- Bonhoeffer, D. (1998). Cartas de amor desde la prisión. Madrid: Trotta.

- Bonhoeffer, D. (1964). Cost of Discipleship. London: SCM Press.

- Buber, M (1995). Yo y Tú. Madrid: Caparrós.

- Crawshaw, R. et al. (1995). Patient-Physician Covenant. Journal of American Medical Association, $273, \mathrm{I} 553$.

- Danzinger, S., Levav, J., Avnaim-Pesso, L. (2OII). Extraneous factors in judicial decisions. Proceedings of the National Academy of Sciences, Published online before print April n, $20 \mathrm{OII}$.

- Donabedian, A. (1989). The End Results of Health Care: Ernest Codman's Contributions to Quality Assessment and Beyond. The Milkbank Quaterly, 67, 233-256.

-Enríquez, E. J. (I595). Retrato del perfecto médico. Salamanca: Juan y Andres Renaut.

- Hilfiker, D. (1984). Facing our Mistakes. The New Englad Journal of Medicine, 310, II8I22.

- Hilfiker, D. (I989) Second Opinion, obtenido el 20 de abril de 201 desde http://www.accessmylibrary.com/article-IGI-7535088/facing-brokeness-interview-david. html

- Hojat, M., Gonnella, J. S. Nasca, T. J., Mangione, S., Vergare, M., Magee, M. (2002) Physician Empathy: Definition, Components, Measurement, and Relatioship to Gender and Specialty. American Journal of Psychiatry, I59, I563-I569.

- Kohn, L. T. Corrigan, J. M. Y Donalson, M. S. Ed. (200I). To err is human. Building a Safer Health System. Committee on Quality of Health Care in America. Institute of Medicine. Washington D.C.: National Academy Press. 
- Lee, L. (2OII). El retrato de un buen médico. Medicina, Societat i Literatura. San Feliu de Guixols, España.

- Localio, A. R. et al. I99I. Relation between Malpractice Claims and Adverse Events Due to Negligence - Results of the Harvard Medical Practice Study III. New England Journal of Medicine, 325, 245-25I.

- Magris, C. (2OII). Discurso de investidura como Doctor Honoris Causa. Barcelona: Universitat de Barcelona.

- Marion, R. (1992). Learning to Play God. New York: Addison-Wesley.

- Miranda, A. de (1983). Diálogo del perfecto médico. Manuel E. Mingote Muñiz (Ed.) Madrid: Editora Nacional.

- Montaigne, M. De (2003). Ensayos completos. Barcelona: Cátedra.

- National Confidential Enquiry into Patient Outcome \& Death. Obtenido el 20 de abril de 2OII desde http://www.ncepod.org.uk/

- Oriol, A. (20Io). El profesionalismo: asignatura pendiente del sistema educativo médico. EIDON, 33, 52-57.

- Pope, A. (1858). The Poetical Works of Alexander Pope. London: Henry G. Bohn.

- Selzer, R. (I996). Letters to a Young Doctor. San Diego: Harcourt Brace \& Company.

- Séneca. (1986). Epístolas Morales a Lucilio. Madrid: Gredos.

- Stern, D. T. Ed. (20o6). Measuring Medical Professionalism. New York: Oxford University Press.

- The Program in Narrative Medicine. College of Physicians and Surgeons, Columbia University. Obtenido el 20 de abril de $20 I$ desde http:/ / www.narrativemedicine.org/

- Vidmar, N. (2OII). The psychology of trial judging. Current Directions in Psychological Sciences, 20, 58-62.

-Wilder, T. (1928). The Angel That Troubled the Waters. London: Longmans, Green and Co. 